\title{
磷影响陆地生态系统碳循环过程及模型表达方法
}

\author{
黄 玫 ${ }^{*}$ 王 娜 ${ }^{1,2}$ 王昭生 $^{1}$ 巩 贺 ${ }^{1}$
}

${ }^{1}$ 中国科学院地理科学与资源研究所生态网络观测与模拟重点实验室, 北京 $100101{ }^{2}{ }^{2}$ 中国科学院大学, 北京 100049

摘 要 全球气候变暖已大大改变了陆地植物碳吸收能力, 提高了全球植被净初级生产力。随着气候变暖的加剧, 磷对植物 生长的限制作用逐渐显现且不断增强, 磷影响陆地生态系统碳循环的机理和模型研究已成为研究热点。该文系统分析了磷影 响陆地生态系统碳循环的相关机理以及模型对相关过程的定量化表达方法。综合对比分析了国际上的CarnegieAmes-Stanford Approach-CNP (CASA-CNP)、Community Land Model-CNP (CLM-CNP)和Jena Scheme for Biosphere-Atmosphere Coupling in Hamburg-CNP (JSBACH-CNP) 等碳、氮、磷耦合模型中磷影响植物光合作用与同化物分配过程、植物对磷的吸收 过程、土壤中磷的转化过程以及生态系统磷输入与输出等过程的相关数学表达方法, 指出了模型算法的局限与不确定性以及 未来模型发展与改进的方向。同时综合对比分析了CASA-CNP、CLM-CNP、JSBACH-CNP模型的基本特征, 总结了磷循环 模型的建模方法, 为未来开展磷影响陆地生态系统碳循环的模型模拟研究提供了借鉴方法与参考思路。

关键词 磷限制; 陆地生态系统; 碳循环; 过程模型; 气候变化

黄玫, 王娜, 王昭生, 巩贺 (2019). 磷影响陆地生态系统碳循环过程及模型表达方法. 植物生态学报, 43, 471-481. DOI: 10.17521/cjpe.2019.0021

\section{Modeling phosphorus effects on the carbon cycle in terrestrial ecosystems}

\author{
HUANG Mei ${ }^{1 *}$, WANG Na ${ }^{1,2}$, WANG Zhao-Sheng ${ }^{1}$, and GONG He ${ }^{1}$ \\ ${ }^{1}$ Key Laboratory of Ecosystem Network Observation and Modeling, Institute of Geographic Sciences and Natural Resources Research, Chinese Academy of \\ Sciences, Beijing 100101, China; and ${ }^{2}$ University of Chinese Academy of Sciences, Beijing 100049, China
}

\begin{abstract}
Climate warming has significantly alerted the terrestrial carbon dynamics, resulting in enhanced vegetation productivity, especially in the northern hemisphere. However, most of the prior modeling studies have neglected the effects of nutrient availability, such as the phosphorus limitation, on carbon processes, which potentially leads to an overestimation of the capacity of terrestrial ecosystems to sequester additional carbon. Here, we reviewed recent progress in phosphorus limitation and its interactions with carbon dynamics in the context of climate change, with a focus on the process-based modeling approach. We comparatively analyzed quantitative representations of phosphorus-associated biological processes in some models (i.e., Carnegie-Ames-Stanford Approach (CASA), Community Land Model (CLM), and Jena Scheme for Biosphere-Atmosphere Coupling in Hamburg $(\mathrm{JSBACH})$ ), such as photosynthesis and distribution of assimilates, phosphorus uptake by plants, the transformation of phosphorus pools in soil, phosphorus inputs and outputs, etc. We also discussed the key characteristics of these models and summarized the mathematical representations of the terrestrial phosphorus cycle. In addition, we identified and discussed the limitations, uncertainties and future needs in process-based modeling in terms of nutrient and carbon dynamics. Our study highlighted the importance of including phosphorus limitation in regional carbon estimation and provided deep insights related to biogeochemical modeling at broad scales.
\end{abstract}

Key words phosphorus limitation; terrestrial ecosystem; carbon cycle; process-based modeling; climate change

Huang M, Wang N, Wang ZS, Gong H (2019). Modeling phosphorus effects on the carbon cycle in terrestrial ecosystems. Chinese Journal of Plant Ecology, 43, 471-481. DOI: 10.17521/cjpe.2019.0021

磷 $(\mathrm{P})$ 是植物体内有机物的组成元素, 广泛参与 植物有机体的代谢过程(刘超等, 2012), 与氮(N) 一
起通过影响植物的光合作用、呼吸作用和土壤有机 质分解等一系列过程实现与碳氮循环过程的耦合

收稿日期Received: 2019-01-22 接受日期Accepted: 2019-04-02

基金项目: 国家自然科学基金(41671101)和中国科学院 A类战略性先导科技专项(XDA23100202)。Supported by the National Natural Science Foundation of China (41671101), and the Strategic Priority Research Program for the Chinese Academy of Sciences (XDA23100202).

*E-mail: huangm@igsnrr.ac.cn 
(李雷等, 2013; 卢少勇等, 2016)。在地史时期, 生物 圈的活动形成了磷在全球范围内的大循环, 磷的循 环与转化在重建气候、环境、生态以及全球变化的 长期反馈机制中有着重要的作用(Föllmi，1996)。一 方面, 地球生命从起源开始就依赖磷, 磷是所有生 命形式的基本元素, 参与生物体的遗传物质与能量 物质的转化, 是构成许多生命大分子(核酸、磷脂、 二磷酸腺苷(ADP)、三磷酸腺苷(ATP)等)的关键元素, 在整个生态系统内能量的咜存、迁移、转化过程中 发挥着重要的作用(Pierrou, 1976; 赵琼和曾德慧, 2005; Achat et al., 2009; Wang et al., 2010)。另一方面, 众多研究表明磷的有效性强烈地影响着陆地生态系 统的碳循环(de Graaff et al., 2006; Norby et al., 2010; Fernández-Martínez et al., 2014): 光合作用产生的糖 类如果不经过磷酸化, 碳的固定则是无效的; 磷也 会加强光合作用过程中碳水化合物的合成与运输; 在植物与土壤中, 磷与碳、氮的化学计量关系不仅 会影响着植物体内碳的积累与分配(周正虎和王传 宽, 2016), 还决定着全球陆地生态系统功能以及碳 的源与汇(Aerts \& Chapin III, 2000; van Wijk et al., 2003; Wan et al., 2005)。

近年来, 随着全球变暖的加剧以及农田生态系 统中磷肥的施入, 全球范围内植被净初级生产力普 遍提高, 磷对植物生长的限制性作用逐渐加强(Goll et al., 2012; Sun et al., 2017)。因此, 能模拟磷循环的 生物地球化学模型对于评估与预测全球碳循环至关 重要。研究表明, 生物地球化学模型如果不考虑磷 限制因素则可能高估植被的碳吸收(Zhang et al., 2014; Reed et al., 2015)。虽然早在2000年初开始出 现模拟磷影响碳循环的模型(Wang et al., 2007), 但 是目前国际上只有少数生物地球化学模型能够模拟 磷循环。因此, 系统总结磷影响陆地生态系统碳循 环过程及其模型表达方法, 一方面能提高我们对该 领域研究态势和趋势的认识, 另一方面对我国生物 地球化学模型的发展具有指导意义。

Carnegie-Ames-Stanford Approach-CNP (CASACNP)、Jena Scheme for Biosphere-Atmosphere Coupling in Hamburg-CNP (JSBACH-CNP)、Community Land Model-CNP (CLM-CNP)模型是目前国际上主 流的能模拟碳、氮、磷循环的生物地球化学模型。 Wang等(2010)基于CASA-CNP模型模拟了全球陆地 生态系统中碳、氮、磷的空间分布, 发现磷的限制
使得热带地区植被净初级生产力减少了 $20 \%$ 。Goll 等(2012)基于 JSBACH-CNP模型模拟评估了磷对 $\mathrm{CO}_{2}$ 浓度升高引起的陆地生态系统碳吸收的影响, 发现磷限制使得全球碳吸收减少了 $16 \%$ 。Yang等 (2014)基于CLM-CNP模型模拟得出热带与温带森 林 1970-1999年间的磷吸收速率为 0.18-0.71 $\mathrm{mg} \cdot \mathrm{m}^{-2} \cdot \mathrm{a}^{-1}$ 。上述过程模型在模拟评估磷对于陆地生 态系统碳循环的影响方面取得了较大的进展。本文 在分析磷参与生态系统碳循环的过程与机理的基础 上, 系统总结了JSBACH-CNP、CASA-CNP、CLMCNP模型中磷在生态系统中循环转化的定量表达方 法, 提出了模型的构建原则和框架, 以期为未来发 展与改进与磷相关的生物地球化学模型提供参考。

\section{1 磷在土壤中的循环过程机理与模型表达}

\section{1 磷输入生态系统过程的机理与模型表达}

大量的实验研究表明磷主要通过风化、沉降、 地表径流等方式输入陆地生态系统, 其中, 风化磷 是由生物和有机磷酸盐通过存储转化而产生的磷的 释放, 土壤的风化基面是磷酸盐暂时的存储库, 岩 性、土壤发育程度、径流、气温、降水、植被类型 等是影响磷风化通量大小的主要因子(Goll et al., 2014; Hartmann et al., 2014)。虽然在某些地区沉降 是磷输入生态系统的重要方式, 但通过沉降方式输 入生态系统的磷可利用性相对较小(Peñuelas et al., 2014), 影响磷沉降通量大小的因子相对复杂, 因此 对其机理的研究还有较大的不确定性。有研究表明 以沉降方式输入生态系统的磷通量与以风化方式输 入的磷通量的量级大致相当(Wang et al., 2015)。径 流是磷输入生态系统的途径之一, 但由于其输入数 量相对较小, 对其机理的研究结果也具有很大的不 确定性。

基于对CASA-CNP、CLM-CNP、JSBACH-CNP 等模型的综合分析, 磷输入生态系统的过程一般量 化表达为风化、沉降、径流等主要磷输入源与其他 可能磷输入源 $\left(F_{\mathrm{P}, \text { others }}\right)$ 之和, 其表达式如下:

$$
P_{\text {input }}=F_{\mathrm{P}, \text { dep }}+F_{\mathrm{P} \text {,wea }}+F_{\mathrm{P} \text {,run }}+F_{\mathrm{P} \text {,others }}
$$

式中, $P_{\text {input }}$ 表示单位时间内输入土壤系统中磷的量, $F_{\mathrm{P}, \text { dep }}$ 为单位时间内沉降的磷通量, $F_{\mathrm{P}, \mathrm{wea}}$ 为单位时间 风化的磷通量, $F_{\mathrm{P}, \text { run }}$ 为单位时间通过地表径流进入 生态系统的磷通量, 单位均为 $\mathrm{mg} \cdot \mathrm{m}^{-2} \cdot \mathrm{a}^{-1}$ 。其中, 通过 沉降输入的磷通量取值为 $0.0007 \mathrm{Gt} \cdot \mathrm{a}^{-1}$ (Mahowald 
et al., 2008), 风化输入的磷通量大多数按照土壤的 风化程度取不同数值, 数值范围在 0.003-0.05 $\mathrm{g} \cdot \mathrm{m}^{-2} \cdot \mathrm{a}^{-1}$ 之间(Newman, 1995), 通过径流输入的磷 通量约为 $0.014 \mathrm{Gt} \cdot \mathrm{a}^{-1}$ (Smil, 2000)。而且研究也表明, 在大于 100 年的时间尺度上, 生态系统中通过风化、 沉降、径流等产生的磷输入量与淋溶流失等磷输出 量是平衡的(Wang et al., 2010)。

研究表明, 人类活动对生态系统的磷输入已产 生了重要影响, 例如在农田生态系统中施用磷肥、 草地生态系统中载畜量的变化等也会对 $P_{\text {input }}$ 产生影 响, 但是施肥输入的不确定性相对较高, 难以量化。 目前的模型都未考虑人类活动对磷输入的影响, 这 将是未来的生物地球化学模型的研究重点(董晓兵 等, 2014; 王芳等, 2014; 严雄风等, 2016)。

\section{2 植物根对磷的吸收过程的机理与模型表达}

根是植物体从土壤中吸收磷的重要器官, 植物 根对磷的获取能力影响着植物体生长发育的全过程 (Ushio et al., 2015; Battini et al., 2017), 而影响植物 根部对磷吸收的主要因素分为两类, 一类为植物体 生理特征, 如根系的形态(廖红等, 2001)、根的结构 (严小龙等, 2000)、根毛(Jungk, 2001)等均影响根部 吸收速率(Zhang et al., 2016); 另一类为根部土壤环 境, 如土壤中磷酸酶(酸性磷酸酶APA)的含量、微生 物的丰富度(Spohn et al., 2018)、菌群、水分(Deepika \& Kothamasi, 2015)等。同时, 不同的物种与不同的 气候环境下根部的磷吸收速率也存在差异。总体而 言, 植物可以通过根系的生长、形成根毛及菌根等 方式提高土壤磷的利用效率。

目前的生物地球化学模型模拟植物根对磷的吸 收, 无法全部量化上述影响因子。模拟量化植物根 对磷的吸收, 主要综合考虑植物的磷需求量与土壤 中溶解磷的含量。如果土壤中的溶解磷的含量大到 能满足植物的磷需求, 则植物根会按需求吸收; 如 果土壤中溶解磷的量小于植物的磷需求量, 则植物 根部磷吸收通量是土壤中溶解磷的量与磷限制因子 的乘积。通过对CASA-CNP、CLM-CNP、JSBACH$\mathrm{CNP}$ 等模型的总结分析得出, 根部磷吸收通量 $P_{\mathrm{r}, \mathrm{sorb}}$ 是吸收系数 $a_{\mathrm{P}, \mathrm{i}}$ 与可供根部吸收的磷通量 $F_{\mathrm{P}, \mathrm{up}}$ 的乘积, 其通用表达式为:

$$
P_{\mathrm{r}, \text { sorb }}=a_{\mathrm{P}, i} \times F_{\mathrm{P}, \text { up }}
$$

在CASA-CNP模型中, 吸收系数 $a_{\mathrm{P}, i}$ 取固定的数 值 0.9 , 而在CLM-CNP模型中, 吸收系数 $a_{\mathrm{P}, i}$ 不再是
一个确定的数值, 而是由经验常数 $K_{\mathrm{s}} 、 S_{\max }$ 和土壤中 每平方米溶解磷的量 $\left(P_{\mathrm{so}}\right)$ 构成的函数, 其具体表达 式为:

$$
a_{\mathrm{P}, i}=\frac{s_{\max } \times k_{\mathrm{s}}}{\left(P_{\mathrm{sol}}+k_{\mathrm{s}}\right)^{2} \times\left(s_{\max }+k_{\mathrm{s}}\right)}
$$

式中, $k_{\mathrm{s}} 、 s_{\text {max }}$ 为经验常数, 在该模型的实验样地 中分别取值为 10 和 0.00035 。

在JSBACH-CNP模型中, 吸收系数 $a_{\mathrm{P}, i}$ 的表达式 为:

$$
a_{\mathrm{P}, i}=\left(1+\frac{s_{\mathrm{max}} \times k_{\mathrm{s}}}{\left(P_{1}+k_{\mathrm{s}}\right)^{2}}\right)^{-1}
$$

式中, $s_{\max }$ 是最大的吸附磷的数量; $k_{\mathrm{s}}$ 是经验常数; $P_{1}$ 为活性磷库的大小。

在未来的模型研究中, 建议改变对 $a_{\mathrm{p}, i}$ 统一赋值 的方式，可以着眼于从不同植被功能型的根系结 构、土壤环境、根系分泌物等方面进行细化、量化、 赋值, 以进一步完善吸收系数 $a_{\mathrm{p}, i}$ 的函数构成, 减小 $a_{\mathrm{p}, \text { i }}$ 数值的不确定性。

\section{3 根部磷吸收来源——活性磷库动态变化过程 的机理与模型表达}

活性磷(不稳定磷, labile P) 是植物可吸收及利 用磷的重要来源, 也是土壤的核心磷库。Johnson等 (2003)认为土壤中的活性磷的存量要远远大于植被 的需求量, 活性磷库的动态变化过程是土壤中磷循 环的核心过程。磷主要由根、茎、叶等凋落物通过 矿化以及动植物残体生物矿化、次生矿物磷的解吸 附、与土壤中溶解磷的交换等主要过程进入活性磷 库, 以根部吸收、矿物颗粒吸附、淋溶流失等过程 从生态系统输出。

虽然CASA-CNP、CLM-CNP、JSBACH-CNP 对活性磷通量的变化表达方法各异, 但综合来看, 活性磷库 $\left(P_{\text {labile }}\right)$ 的通量变化与生态系统外部输入的 磷通量 $\left(P_{\text {input }}\right)$ 、生态系统内部输入的磷通量 $\left(P_{\text {input,in }}\right)$ 、 活性磷库输出的磷通量 $\left(P_{\text {output }}\right)$ 以及限制因子函数 $\left(f_{\mathrm{lim}}\right)$ 有关, 其动态变化为输入的磷通量与输出的磷 通量之间的差与限制因子的乘积:

$$
\frac{d P_{\text {labile }}}{d t}=f_{\text {lim }} \times\left(P_{\text {input }}+P_{\text {input, in }}-P_{\text {output }}\right)
$$

生态系统内部输入的磷通量 $P_{\text {input,in }}$ 主要来源于 土壤有机质库中生物化学磷和生物库中生物磷的矿 化，因此其通用表达式为: 


$$
P_{\text {input,in }}=F_{\mathrm{P} \text {,tase }}+F_{\mathrm{P}, \text { net }}
$$

式中, $F_{\mathrm{P}, \text { tase }}$ 为生物化学磷的矿化通量, $F_{\mathrm{P}, \text { net }}$ 是单位 时间生物磷的矿化通量, 单位均为 $\mathrm{mg} \cdot \mathrm{m}^{-2} \cdot \mathrm{a}^{-1}$ 。

活性磷库主要通过植物吸收、淋溶流失、吸附 等方式输出, 因此其输出通量的通用表达式为:

$$
P_{\text {output }}=F_{\mathrm{P}, \text { take }}+F_{\mathrm{P} \text {, leaching }}+F_{\mathrm{P}, \text { sorb }}+F_{\mathrm{P}, \text { others }}
$$

式中, $F_{\mathrm{P}, \text { take }}$ 是植物吸收的磷通量, $F_{\mathrm{P}, \text { leaching 是淋溶流 }}$

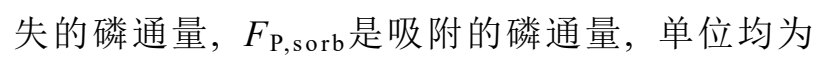
$\mathrm{mg} \cdot \mathrm{m}^{-2} \cdot \mathrm{a}^{-1} ; F_{\mathrm{P}, \text { others }}$ 表示其他可能影响活性磷库中磷 输出过程的表达式。

由于影响活性磷库变化的因子相对复杂, 不同 模型对活性磷库动态变化的表达在上述一般性描述 的基础上存在差异。如在CLM-CNP模型中, 分别量 化了生物化学矿化磷的通量输入以及生物磷的矿化 输入, 但是在整个计算过程中设置的限制性参数相 对较少, 具体过程见下文分析; 而在JSBACH-CNP 模型中, 没有对生物磷的矿化通量进行计算, 而且 在对生态系统输出的磷通量量化过程中既包括植物 对磷的吸收, 也包括了微生物的磷需求, 其他两个 模型目前暂未量化微生物对磷的需求量。另外, 限 制因子函数 $f_{\mathrm{lim}}$ 与生态系统类型、环境因素等有密切 的关系, 其量化仍然存在很大的不确定性。综合而 言, 上述三个模型对不稳定磷动态变化过程的模拟 量化较为完整, 与机理表达过程一致性较高, 但是 量化过程的精确化与精准性仍有提高的空间。在未 来的模拟过程中, 对于 $P_{\text {input,in }}$ 的量化过程可以考虑 按照不同的矿化速率来量化矿化通量的大小, $P_{\text {output }}$ 的量化过程中对于微生物的磷需求与限制因子的函 数构成均有待优化。

\section{4 有机磷矿化过程的机理及模型表达}

土壤中的有机磷只有经过矿化后才能被植物吸 收利用, 有机磷的矿化过程可以源源不断地为植物 提供新的磷来源, 有机磷的矿化过程包括生物矿化 过程和生物化学矿化过程两部分, 其中生物化学矿 化过程是有机磷矿化的主导过程。土壤中矿化的磷 通量大小不仅取决于环境因子, 还取决于生物因子 (Niu et al., 2013)。土壤中有机磷的矿化速率与有机 碳的含量也有一定的关系, 很多研究发现有机碳的 含量与有机磷的矿化速率呈显著的正相关关系 $(\mathrm{Scott}$ \& Condron, 2005; Thomas et al., 2006)。另外, 土壤 的温度、湿度、孔隙度等理化性质影响着有机磷矿
化通量的大小, 如Campbell和Racz (1975)研究发现 在含水量较高的土壤中有机磷的矿化较强, 同时也 有研究表明土壤温度升高可以加快土壤中有机磷的 矿化速率(Turner et al., 2003)。土壤中的酶是有机磷 矿化过程的重要催化剂, 如磷酸酶是有机磷矿化的 首要催化剂, 可以促进土壤中有机物释放无机磷, 其活性的高低影响着土壤中有机磷的分解转化 (Wang et al., 2010)。

基于上述机理, 模型归纳得出有机磷矿化通量 $\left(F_{\mathrm{m}, \mathrm{P}}\right)$ 由生物矿化通量 $\left(F_{\mathrm{bi}}\right)$ 与生物化学矿化通量 $\left(F_{\mathrm{bc}}\right)$ 构成:

$$
F_{\mathrm{m}, \mathrm{P}}=F_{\mathrm{bi}}+F_{\mathrm{bc}}
$$

CASA-CNP、CLM-CNP、JSBACH-CNP三个生 物地球化学模型均对 $F_{\mathrm{bc}}$ 进行了定量模拟, 但是由于 生物矿化过程的量化相对复杂, 仅有CLM-CNP模 型对 $F_{\mathrm{b}}$ 进行了定量表达。 $F_{\mathrm{bc}}$ 的通量大小是土壤有机 质库中磷的总量 $P_{\mathrm{SOM}}$ 与通量系数 $r_{\mathrm{bc}}$ 的乘积:

$$
F_{\mathrm{bc}}=P_{\mathrm{SOM}} \times r_{\mathrm{bc}}
$$

通量系数 $r_{\mathrm{bc}}$ 是决定模型中磷矿化通量精准程度 的关键因子, 但是影响 $r_{\mathrm{bc}}$ 的因子众多, 因此各模型 在 $r_{\mathrm{bc}}$ 的量化表达上并不完全相同。如在CASA-CNP 模型中 $r_{\mathrm{bc}}$ 的表达式为:

$$
r_{\mathrm{bc}}=\frac{\lambda_{\mathrm{P}, \text { up }}-\lambda_{\mathrm{P}, \text { tase }}}{\lambda_{\mathrm{P}, \text { up }}-\lambda_{\mathrm{P}, \text { tase }}+k_{\mathrm{P}, \text { tase }}} \times v_{\mathrm{P}, \text { max }}
$$

式中, $v_{\mathrm{P}, \text { max }}$ 为最大矿化速率; $\lambda_{\mathrm{P}, \mathrm{up}}$ 是磷吸收的氮消耗, 即每吸收 $1 \mathrm{~g}$ P 消耗 $\mathrm{N}$ 的数量, 在热带生物群取值为 $40 \mathrm{~g} \cdot \mathrm{g}^{-1}$, 其他地区取值为 $25 \mathrm{~g} \cdot \mathrm{g}^{-1} ; K_{\mathrm{P}, \text { tase }}$ 是磷酸盐生 产的氮消耗, 即每生产 $1 \mathrm{~g}$ 磷酸盐消耗 $\mathrm{N}$ 的数量, 取

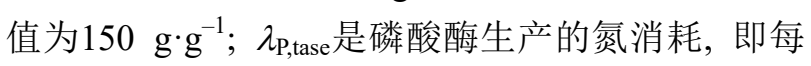
生产 $1 \mathrm{~g}$ 磷酸酶所消耗 $\mathrm{N}$ 的数量, 取值为 $15 \mathrm{~g} \cdot \mathrm{g}^{-1}$ 。

CLM-CNP模型对 $r_{\mathrm{bc}}$ 的考虑更为全面, 同时考 虑了氮和磷需求的限制因子, 其表达式为:

$$
r_{\mathrm{bc}}=k_{\mathrm{bc}} \times f_{\mathrm{lim}}^{\mathrm{N}} \times\left(1-e^{\tau_{\mathrm{bc}} \times\left(1-f_{\mathrm{lim}}^{\mathrm{P}}\right)}\right)
$$

式中, $k_{\mathrm{bc}}$ 是生物化学矿化速率; $\tau_{\mathrm{bc}}$ 是生化矿化的标 度因子; $f_{\lim }^{\mathrm{N}} 、 f_{\lim }^{\mathrm{P}}$ 表示矿化需求过程中的氮、 磷限制因子。

上述公式对影响磷矿化的通量大小的机理有了 相对完整的表述, 但是在实际的生态系统中, 土壤 中矿化的磷通量大小更为复杂, 不仅仅取决于环境 因子, 还取决于生物因子(Niu et al., 2013), 在未来 的研究中可以通过以下几个方面提高表达式的精准 
性, 如将实验测量与模型模拟相结合, 完善土壤理 化性质数据库, 完善 $r_{\mathrm{bc}}$ 的量化表达式, 加入土壤有 机碳含量、土壤温度、磷酸酶活性等相关参数, 使 模拟过程与机理更加贴近现实。

\section{5 磷在植物器官内部分配过程的机理与模型 表达}

植物体根部吸收磷后，按照一定的比例输送、 分配给根、茎、叶器官。模型对植物体磷的分配的 定量描述主要依据根、茎、叶中的碳、氮、磷的化 学计量关系来计算(Yang et al., 2014)。植物体各器官 获得的磷通量 $\left(F_{\mathrm{u}, \mathrm{i}}\right)$ 的通用表达式为:

$$
F_{\mathrm{u}, i}=\mu_{i} \times r_{\mathrm{P}, i} \times P_{i}
$$

式中, $r_{\mathrm{P}, ~}$ 对应库 $i$ (茎、叶)的磷吸收系数; $\mu_{i}$ 对应库的 周转速率, 不同模型取值不同; $P_{i}$ 对应库中的磷含 量, 单位为 $m g$ 。磷吸收系数 $r_{\mathrm{P}, i}$ 主要采用两种方式进 行量化计算。一种是以CASA-CNP模型为代表的简 单分配系数, $r_{\mathrm{P}, i}$ 的数值分别为 $0.5 、 0.9$ 。另一种以 CLM-CNP、JSBACH-CNP模型为代表的与植物器官 对应的碳分配的比例相关, 其中JSBACH-CNP模型 设置为根、茎、叶等库, 而CLM-CNP库的设置较为 细化, 分为细根、粗根、新茎、新叶等。

根、茎、叶吸收磷后, 将以调落物的形式输入 到对应的调落物库中, 根、茎、叶调落物库是连接 植物库与土壤有机质库的中转站, 其磷的输入源为 根、茎、叶各器官的调落物, 其磷主要输出到对应 的土壤有机质库。因此调落物库磷动态变化的量化 为根、茎、叶输入的磷与输出到土壤有机质库磷的 差值, 输入量大小取决于根、茎、叶周转速率的快 慢与碳、磷比值的高低, 其输出量取决于凋落物库 的分解速率与周转速率。

\section{6 磷输出生态系统过程的机理及模型表达}

磷主要通过地表径流沿着土壤剖面, 通过淋 溶、侵蚀、吸附以及向闭蓄态磷库转化等方式输出 生态系统(Pote \& Daniel, 1996; McGroddy et al., 2004; Mahowald et al., 2008)。影响磷输出生态系统 过程的机理相对复杂, 土壤的磷含量、植被覆盖状 况、土壤类型、土壤微生物以及气候条件等都是影 响磷流失的因子。活性磷库的大小以及径流量是影 响磷以淋溶方式输出生态系统的主要因子 (Mahowald et al., 2008), 同时气候与环境因素对以 淋溶、侵蚀等方式流失的磷具有影响(McGroddy et al., 2004), 但是气候与环境因子量化难度较高。
CASA-CNP、CLM-CNP和JSBACH-CNP等模型

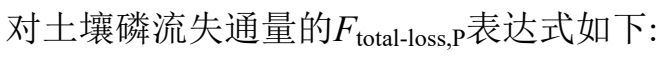

$$
F_{\text {total-loss,P }}=F_{\mathrm{P}, \text { leaching }}+F_{\text {ocl }}
$$

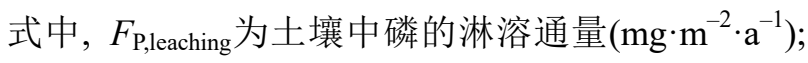
$F_{\mathrm{ocl}}$ 表示从次生矿物磷库转为闭蓄态磷的通量

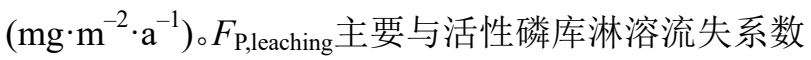
与活性磷库中磷含量有关, 可表达如下:

$$
F_{\mathrm{P}, \text { leaching }}=f_{\mathrm{P}} \times P_{\text {labile }}
$$

式中, $f_{\mathrm{P}}$ 为活性磷库中磷淋溶流失的系数, 普遍取值

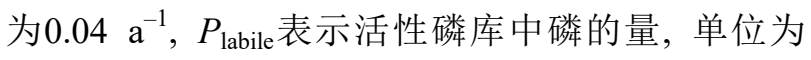
$\mathrm{g} \cdot \mathrm{m}^{-2}$ 。

从次生矿物磷库转为闭蓄态磷的速率通量 $F_{\mathrm{ocl}}$ 具体表达式为:

$$
F_{\mathrm{ocl}}=r_{\mathrm{ocl}} \times P_{\mathrm{sec}}
$$

式中, $r_{\mathrm{ocl}}$ 表示从次生矿物磷转为闭蓄态磷的通量系 数; $P_{\mathrm{sec}}$ 表示次生矿物磷库中磷的量 $\left(\mathrm{g} \cdot \mathrm{m}^{-2}\right)$ 。

虽然磷输出陆地生态系统的过程和气候与环境 因子有关, 但是目前的模型在量化此过程中并未考 虑气候与环境因子对磷输出过程的影响。在百年以 上的时间尺度上, 磷输入与输出陆地生态系统的量 是平衡的。但是要在较短时间内进行模拟, 应更加 全面地考虑影响磷输出陆地生态系统的气候与环境 因子以提高模拟的准确性。目前的模型均假设吸附 与解吸附过程处于相对平衡状态, 因此暂未量化该 过程。

\section{2 磷影响植物体碳循环过程的机理与模型 表达方法}

\section{1 磷影响光合作用过程的机理与模型表达}

土壤与叶片中的磷均影响植物光合作用过程。 实验表明, 土壤中以无机磷为主导的多种形态的磷, 不仅会直接影响光合作用的能力和速率, 而且也会 间接地影响着植物叶片中磷的浓度(Ellsworth et al., 2015)。一方面, 土壤中的无机磷可以被植物体吸收, 其不仅充当光合作用的基底物，而且是形成光合作 用的生物分子的重要元素(Reich \& Oleksy, 2004)。 另一方面, 土壤中的无机磷影响着二磷酸核酮糖羧 化酶的形成, 从而影响着光合作用的速率(Norby et al., 2015, 2017)。同样, 植物叶片中磷的浓度与植物 叶片光合速率的相关性十分显著, 如果叶片中磷供 应不足会影响磷酸甘油酸的还原和再生(Fredeen et $a l ., 2018)$, 降低叶绿体间质的 $\mathrm{pH}$ 值以及参与卡尔文 
循环的各种酶的活性, 最终影响光合作用的强度、 羊夋化速率等(Lloyd et al., 2001; Wang et al., 2017)。

模型关于磷对光合作用的限制往往与氮限制结 合起来, 取二者最小值作为营养元素的限制。一般 步骤为首先要计算出一个不受氮、磷影响时的最大 光合速率 $F_{\mathrm{c}, \text { max }}$, 而植物实际的光合速率 $F_{\mathrm{c}}$ 为 $F_{\mathrm{c}, \text { max }}$ 叶片磷限制因子 $\left(x_{\mathrm{P}, \text { leaf }}\right) 、$ 叶片氮限制因子 $\left(x_{\mathrm{N}, \text { leaf }}\right)$ 共同 构成的函数, 其表达式为:

$$
F_{\mathrm{c}}=\min \left(x_{\mathrm{P}, \text { leaf }}, x_{\mathrm{N}, \text { leaf }}\right) \times F_{\mathrm{c} \text {, max }}
$$

Lloyd等(2001)研究发现, $x_{\mathrm{P}, \text { leaf }}$ 的响应曲线和 $x_{\mathrm{N}, \text { leaf }}$ 的 响应曲线类似, 这一理论与Ghannoum等(2008)在草 地上开展的相关实验结论相契合, 因此 $x_{\mathrm{P}, \text { leaf }}$ 的算法 与 $x_{\mathrm{N}, \text { leaf }}$ 的算法较为类似。CASA-CNP、CLM-CNP、 JSBACH-CNP等模型主要利用 $x_{\mathrm{P}, \text { leaf }}$ 来量化磷对光合 作用强度的影响，具体表达式为:

$$
x_{\mathrm{P}, \text { leaf }}=\frac{p_{\text {leaf }}}{p_{\text {leaf }}+k_{\mathrm{p}}}
$$

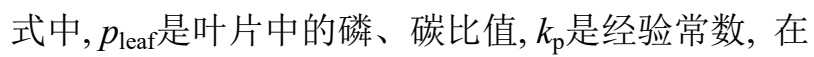
CASA-CNP模型中取值为 $0.0006 \mathrm{~g} \cdot \mathrm{g}^{-1}$ 。在CLMCNP和JSBACH-CNP模型中将 $x_{\mathrm{P}, \text { leaf }}$ 看作是土壤中的 溶解性磷 $P_{\text {soil }}$ 与植物和微生物对磷的需求之比 $P_{\text {demand: }}$

$$
x_{\mathrm{P}, \text { leaf }}=\frac{P_{\text {soil }}}{P_{\text {demand }}}
$$

事实上, 目前生物地球化学模型对磷影响光合 作用过程的量化过程尚未考虑磷与最大羧化速率之 间的显示表达。叶片最大羧化速率是表征植物光合 作用能力的关键参数之一, 对光合速率起着决定性 作用(张彦敏和周广胜, 2012), 但目前相关机理研究 的理论还不成熟。因此未来的发展趋势是, 通过控 制实验揭示磷影响光合作用速率的理论关系, 从而 发展能表达磷影响光合作用的模型。

\section{2 磷影响呼吸作用过程的机理与模型表达}

磷影响植物吸收全过程。叶片中磷浓度与植物 呼吸之间具有较强的相关性, 在热带地区这种相关 性尤为明显。研究发现, 热带森林中叶片磷浓度显 著影响着植物的呼吸作用(Meir et al., 2001; Atkin et al., 2015), Domingues等(2010)在热带地区观测到较 低的叶片磷浓度明显影响叶片暗呼吸(Slot et al., 2014)。另外, Atkin等(2015)发现磷的可利用性还影 响着叶片暗呼吸与氮之间的关系。

磷同样影响土壤呼吸过程。磷有可能通过改变
土壤的生物化学和生理过程来改变土壤呼吸对温度 的敏感性(Jiang et al., 2015)。但目前对磷影响土壤 呼吸作用的机理研究仍然存在较大不确定性(Stitt \& Hurry, 2002; Davidson \& Janssens, 2006; Jiang et $a l ., 2015)$ 。这些不确定性使得当前的生物地球化学 模型不能实现对此过程的独立定量化表达，因此模 拟量化磷对植物和土壤呼吸作用的影响是未来野外 控制实验与模型研究亟待突破的难点(Plaxton \& Podestá, 2006)。

\section{3 磷影响光合作用产物分配过程的机理与模型 表达}

磷对光合作用产物的输送与分配有重要影响。 同化物的分配过程不仅影响着植物的生长, 也会通 过改变光合产物在植物根、茎、叶中的分配比例来 影响调落物的分解速率以及磷的蓄积与分配过程, 从而影响整个碳循环过程(Monteith \& Moss, 1977)。 同样，植物可利用磷的多少也影响着植物同化物的 输送与分配过程，如Bender等(1986)发现当根部有 机磷浓度为原来的 2 倍后, 植物的同化物运输速率 都会显著提高。Ellsworth等(2015)发现长期明显的 无机磷浓度减少将会导致植物组织同化物产生速率 以及分配速率降低。目前的模型还没有磷影响光合 作用产物分配的相关算法, 主要是因为此方面的理 论和机理研究还不成熟。未来需加强此方面的实验 研究, 从而使得生物地球化学模型可以量化此过程。

\section{3 磷影响陆地生态系统碳循环的建模方法}

过程模型量化磷对生态系统碳循环的影响，由 于机理的复杂性与不确定性, 模型量化难度相对较 大。虽然各模型定量表达磷影响陆地生态系统过程 的方法有差异, 但是综合起来看, 模拟磷影响陆地 生态系统碳循环的模型结构如图1所示。一般将陆地 生态系统分为植物库、调落物库和土壤库三个主体 库, 植物库内一般按照根、茎、叶等器官设置磷库, 调落物库主要按照根、茎、叶等不同器官的调落物 类型进行磷库设置, 土壤库依据凋落物分解的有机 质的类型与磷形态设置磷库。磷主要通过风化、沉 降、施肥等方式输入到陆地生态系统, 输入生态系 统中的磷被植物体吸收利用后经矿化、吸附等方式 实现各种不同形态磷之间的循环转换，最终通过淋 溶、风蚀的方式从生态系统输出。模型利用微分方 程量化每个库中磷的动态变化, 结合各组织器官的

www.plant-ecology.com 
碳磷比值量化其对陆地生态系统碳循环的影响。图1 为磷影响陆地生态系统碳循环的结构图, 从中可清 楚地看出磷在植物库、土壤库和调落物库中的循环 转化过程。

CASA-CNP、CLM-CNP和JSBACH-CNP模型在 磷库设置与模拟时间步长等方面的差异如表 1 所示。 从磷库数量来看, CLM-CNP的磷库设置较为细化, 数量为 15 个。CASA-CNP设置的磷库数量为 12 个, JSBACH-CNP模型的磷库设置为 8 个, 最为简化。从 模拟的时间步长看, CASA-CNP和JSBACH-CNP模 型的时间步长为 1 天, CLM-CNP模型的时间步长为 $30 \mathrm{~min}$ 。碳磷比值是模型采用的重要参数, CASACNP与CLM-CNP模型均根据不同植被功能型、不同 植物器官设置不同的碳磷比值参数, 而 JSBACH$\mathrm{CNP}$ 模型则较为简单, 每种植被功能型设置一个碳 磷比值。从矿化过程的模拟来看, CASA-CNP仅量化 了生物化学矿化过程, 而 CLM-CNP和 JSBACH$\mathrm{CNP}$ 模型同时量化了生物矿化过程与生物化学矿化
过程，使得估算的磷通量更为准确。从模型的适应 范围来看, CASA-CNP、JSBACH-CNP模型主要适用 于热带、温带的森林和草原生态系统; CLM-CNP模 型主要适用于热带森林与草原生态系统。从模型的 不完善性来看, CASA-CNP模型没有模拟调落物磷 的生物化学矿化作用, 使得模拟量化的可利用磷的 数量较低; JSBACH-CNP模型对磷循环的模拟相对 简单, 其对碳磷比值的设置也不够精细。CLM-CNP 对磷循环机理的模拟相对完善, 且其积分的时间步 长短，是未来碳、氮、磷模型的发展方向。以上三 个模型都没有模拟碳、氮、磷协同作用与反馈过程, 这是未来模型研究需要着重研究的方向。

\section{4 结论和展望}

本文系统总结了磷影响陆地生态系统碳循环过 程的机理及模型定量化表达方法。重点分析了 CASA-CNP、CLM-CNP、JSBACH-CNP等模型对磷 参与光合作用、同化物分配、植物对磷的吸收以及

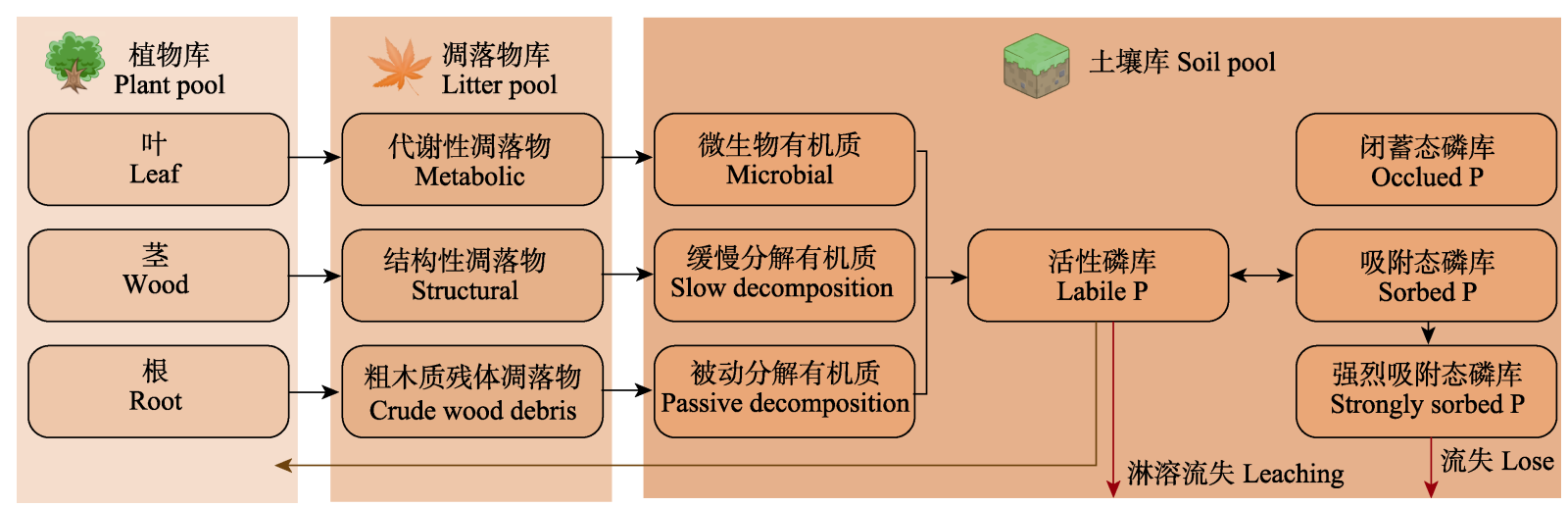

图1 磷影响陆地生态系统碳循环的模型结构图。

Fig. 1 Schematic diagram of phosphorus effects on the terrestrial ecosystem carbon cycles.

表1 CASA-CNP、CLM-CNP和JSBACH-CNP模型的主要构架对比分析

Table 1 Comparison of phosphorus processes in CASA-CNP, CLM-CNP, and JSBACH-CNP

\begin{tabular}{|c|c|c|c|c|c|}
\hline $\begin{array}{l}\text { 模型 } \\
\text { Model }\end{array}$ & $\begin{array}{c}\text { 磷库数量 } \\
\text { Number of } \\
\text { phosphate pools }\end{array}$ & $\begin{array}{l}\text { 时间步长 } \\
\text { Time step }\end{array}$ & $\begin{array}{l}\text { 碳磷比参数 } \\
\text { C:P ratio parameters }\end{array}$ & $\begin{array}{l}\text { 矿化过程模拟 } \\
\text { Simulation of the mineralization } \\
\text { process }\end{array}$ & $\begin{array}{l}\text { 适用范围 } \\
\text { Scope of application }\end{array}$ \\
\hline CASA-CNP & 12 & $1 \mathrm{~d}$ & $\begin{array}{l}\text { 不同植被类型、植物不同器官具有不同 } \\
\text { 的碳磷比 } \\
\text { C:P ratios vary among different organs in } \\
\text { various vegetation types }\end{array}$ & $\begin{array}{l}\text { 只量化了生物化学矿化过程 } \\
\text { Only consider the biochemical } \\
\text { mineralization process }\end{array}$ & $\begin{array}{l}\text { 温带和热带森林生态系统 } \\
\text { 温带和热带草原生态系统 } \\
\text { Temperate and tropical forest } \\
\text { and grassland ecosystems }\end{array}$ \\
\hline CLM-CNP & 15 & $30 \mathrm{~min}$ & $\begin{array}{l}\text { 不同植被类型、植物不同器官具有不同 } \\
\text { 的碳磷比 } \\
\text { C:P ratios vary among different organs in } \\
\text { various vegetation types }\end{array}$ & $\begin{array}{l}\text { 模拟了生物矿化与生物化学矿化 } \\
\text { 两个过程 } \\
\text { Consider both biomineralization and } \\
\text { biochemical mineralization }\end{array}$ & $\begin{array}{l}\text { 热带森林生态系统 } \\
\text { 热带草原生态系统 } \\
\text { Tropical forest and grassland } \\
\text { ecosystems }\end{array}$ \\
\hline JSBACH-CNP & 8 & $1 \mathrm{~d}$ & $\begin{array}{l}\text { 不同植被类型具有不同碳磷比, 但植物 } \\
\text { 不同器官的碳磷比相同 } \\
\text { C:P ratios are the same for organs but vary } \\
\text { among various vegetation types }\end{array}$ & $\begin{array}{l}\text { 模拟了生物矿化与生物化学矿化 } \\
\text { 两个过程 } \\
\text { Consider both biomineralization and } \\
\text { biochemical mineralization }\end{array}$ & $\begin{array}{l}\text { 温带和热带森林生态系统 } \\
\text { 温带和热带草原生态系统 } \\
\text { Temperate and tropical forest } \\
\text { and grassland ecosystems }\end{array}$ \\
\hline
\end{tabular}

CASA-CNP, Carnegie-Ames-Stanford Approach-CNP; JSBACH-CNP, Jena Scheme for Biosphere-Atmosphere Coupling in Hamburg-CNP; CLM-CNP, Community Land Model-CNP. 
磷在土壤中的转化等过程的相关数学表达方法。通 过分析发现模拟磷循环的模型重点量化了磷输入与 输出、磷对光合作用过程、活性磷库动态变化、有 机磷矿化、根部对磷的吸收等过程的影响, 量化过 程基本覆盖了陆地生态系统磷循环的重要过程。

模型对磷输入与输出过程量化的准确性主要取 决于对磷输入源与输出源量化的全面性与精确度。 当前的模型主要量化了风化、沉降、径流等输入源 以及淋溶、侵蚀、吸附、向闭蓄态磷库转化等输出 源, 尚未量化人类活动, 如土地利用变化和施肥等 对陆地生态系统磷输入与输出的影响。未来, 量化 人类活动造成的陆地生态系统磷的输入与输出是生 物地球化学模型的发展方向之一。

机理研究发现, 磷影响植物的光合作用、呼吸 作用、同化物分配等过程。但是由于磷影响呼吸作 用和同化物分配过程的机理研究不确定性较大, 因 此目前的模型尚无磷影响呼吸作用和同化物分配过 程的量化方法。模型对磷影响光合作用的算法都为 隐式算法, 即通过磷限制因子来限制光合作用。机 理研究表明叶片中磷的浓度与羧化速率有很强的相 关性, 因此应该存在磷与羧化速率之间的量化函数 关系式, 但是目前还没有此类的定量关系式。因此 未来在机理研究的基础上, 应进一步完善磷与羧化 速率之间的关系以提高对磷影响光合作用的模拟 能力。

根部磷吸收过程量化的精准性主要取决于根部 吸收系数的函数构成, 当前量化过程考虑的因子尚 不够全面, 未来可考虑加入根的形状等影响因子来 构建根部磷吸收系数。有机磷的矿化过程主要量化 了矿化过程中磷的两大主要通量: 生物矿化磷通量 与生物化学矿化磷通量, 当前的模型较好地反映了 磷矿化过程的机理。不可忽略的是, 理论与实验研 究同时表明未经矿化的有机磷在植物的生长发育过 程中也有重要的作用, 尤其在热带植物的生长发育 过程中发挥着不可忽视的作用, 实验研究表明溶解 于水的有机磷可以直接被作物所吸收利用, 并且用 $\mathrm{NaHCO}_{3}$ 提取的有机磷与植物磷吸收的通量之间呈 显著的相关性(Bowman \& Cole, 1978), 但是目前的 模型都忽略了与有机磷相关的其他生物化学过程。

总之, 目前模拟磷影响碳循环过程的模型的方 法与技术手段还不够成熟, 而且缺乏对碳、氮、磷 协同作用与反馈的模拟。未来的生物地球化学模型
需着力完善磷与碳、氮循环协同及反馈过程的量化, 更加精准地量化与预测磷对于陆地生态系统碳循环 的影响。

\section{参考文献}

Achat DL, Bakker MR, Morel C (2009). Process-based assessment of phosphorus availability in a low phosphorus sorbing forest soil using isotopic dilution methods. Soil Science Society of America Journal, 73, 2131-2142.

Aerts R, Chapin III FS (2000). The mineral nutrition of wild plants revisited: A re-evaluation of processes and patterns. Advances in Ecological Research, 30, 1-67.

Atkin OK, Bloomfield KJ, Reich PB, Tjoelker MG, Asner GP, Bonal D, Bönisch G, Bradford MG, Cernusak LA, Cosio EG (2015). Global variability in leaf respiration in relation to climate, plant functional types and leaf traits. New Phytologist, 206, 614-636.

Battini F, Grønlund M, Agnolucci M, Giovannetti M, Jakobsen I (2017). Facilitation of phosphorus uptake in maize plants by mycorrhizosphere bacteria. Scientific Reports, 7, 4686. DOI: 10.1038/s41598-017-04959-0.

Bender L, Stiebeling B, Neumann KH (1986). Investigations on photosynthesis and assimilate translocation in Daucus carota $\mathrm{L}$. as influenced by a varied phosphorus supply and changes in the endogenous hormonal system following $\mathrm{GA}_{3}$ treatments. Journal of Plant Nutrition and Soil Science, 149, 533-540.

Bowman RA, Cole CV (1978). An exploratory method for fractionation of organic phosphorus from grassland soils. Soil Science, 125, 95-101.

Campbell LB, Racz GJ (1975). Organic and inorganic P content, movement and mineralization of $\mathrm{P}$ in soil beneath a feedlot. Canadian Journal of Soil Science, 55, 457-466.

Davidson EA, Janssens IA (2006). Temperature sensitivity of soil carbon decomposition and feedbacks to climate change. Nature, 440, 165-173.

de Graaff MA, van Groenigen KJ, Six J, Hungate B, van Kessel C (2006). Interactions between plant growth and soil nutrient cycling under elevated $\mathrm{CO}_{2}$ : A meta-analysis. Global Change Biology, 12, 2077-2091.

Deepika S, Kothamasi D (2015). Soil moisture-A regulator of arbuscular mycorrhizal fungal community assembly and symbiotic phosphorus uptake. Mycorrhiza, 25, 67-75.

Domingues TF, Meir P, Feldpausch TR, Saiz G, Veenendaal EM, Schrodt F, Bird M, Djagbletey G, Hien F, Compaore H, Diallo A, Grace J, Lloyd J (2010). Co-limitation of photosynthetic capacity by nitrogen and phosphorus in West Africa woodlands. Plant, Cell \& Environment, 33, 959-980.

Dong XB, Hao MD, Guo SA, Shi XJ, Ma T, Liu GS (2014). The effects of nitrogen fertilizers and phosphate fertilizer

www.plant-ecology.com 
rates on the yield, nutrient uptake and quality of Leymus chinensis. Acta Agrestia Sinica, 22, 1232-1238. [董晓兵, 郝明德, 郭胜安, 石学军, 马甜, 刘公社 (2014). 氮磷 肥配施对羊草干草产量、养分吸收及品质影响. 草地学 报, 22, 1232-1238.]

Ellsworth D, Crous KY, Lambers H, Cooke J (2015). Phosphorus recycling in photorespiration maintains high photosynthetic capacity in woody species. Plant, Cell \& Environment, 38, 1142-1156.

Fernández-Martínez M, Vicca S, Janssens IA, Sardans J, Luyssaert S, Campioli M, Chapin III FS, Ciais P, Malhi Y, Obersteiner M, Papale D, Piao SL, Reichstein M, Rodà F, Peñuelas J (2014). Nutrient availability as the key regulator of global forest carbon balance. Nature Climate Change, 4, 471-476.

Föllmi KB (1996). The phosphorus cycle, phosphogenesis and marine phosphate-rich deposits. Earth-Science Reviews, 40, 55-124.

Fredeen AL, Madhusudana Rao I, Terry N (2018). Influence of phosphorus nutrition on growth and carbon partitioning in Glycine max. Plant Physiology, 89, 225-230.

Ghannoum O (2008). $\mathrm{C}_{4}$ photosynthesis and water stress. Annals of Botany, 103, 635-644.

Goll DS, Brovkin V, Parida BR, Reick CH, Kattge J, Reich PB, van Bodegom PM, Niinemets Ü (2012). Nutrient limitation reduces land carbon uptake in simulations with a model of combined carbon, nitrogen and phosphorus cycling. Biogeosciences, 9, 3547-3569.

Goll DS, Moosdorf N, Hartmann J, Brovkin V (2014). Climate driven changes in chemical weathering and associated phosphorus release since 1850: Implications for the land carbon balance. Geophysical Research Letters, 41, 3553-3558.

Hartmann J, Moosdorf N, Lauerwald R, Hinderer M, West AJ (2014). Global chemical weathering and associated P-release-The role of lithology, temperature and soil properties. Chemical Geology, 363, 145-163.

Jiang J, Guo S, Zhang Y, Liu Q, Wang R, Wang Z, Li N, Li R (2015). Changes in temperature sensitivity of soil respiration in the phases of a three-year crop rotation system. Soil \& Tillage Research, 150, 139-146.

Johnson AH, Frizano J, Vann DR (2003). Biogeochemical implications of labile phosphorus in forest soils determined by the Hedley fractionation procedure. Oecologia, 135, 487-499.

Jungk A (2001). Root hairs and the acquisition of plant nutrients from soil. Journal of Plant Nutrition and Soil Science, 164, 121-129.

Li L, Huang M, Gu FX, Zhang L (2013). The modeling algorithms for the effects of nitrogen on terrestrial vegetation carbon cycle process. Journal of Natural Resources, 28, 2012-2022. [李雷, 黄玫, 顾峰雪, 张黎 (2013). 氮素影
响陆地生态系统碳循环过程的模型表达方法. 自然资 源学报, 28, 2012-2022.]

Liao H, Ge ZY, Yan XL (2001). Ideal root architecture for phosphorus absorption in plants under water-phosphorus coupling stress: Simulation and application. Chinese Science Bulletin, 46, 641-646. [廖红, 戈振扬, 严小龙 (2001). 水磷耦合胁迫下植物磷吸收的理想根构型: 模 拟与应用. 科学通报, 46, 641-646.]

Liu C, Wang Y, Wang N, Wang GX (2012). Advances research in plant nitrogen, phosphorus and stoichiometry in terrestrial ecosystems: A review. Chinese Journal of Plant Ecology, 36，1205-1216. [刘超, 王洋, 王楠, 王根轩 (2012). 陆地生态系统植被氮磷化学计量研究进展. 植 物生态学报, 36, 1205-1216.]

Lloyd J, Bird MI, Veenendaal EM, Kruijt B (2001). Global Biogeochemical Cycles in the Climate System. Academic Press, London. 96-144.

Lu SY, Liu XX, Li K, Gao SH, Jia JL, Yang GY (2016). Phosphorus removal by ecological planting tank treating rainwater runoff. Chinese Journal of Environmental Engineering, 10, 3434-3438. [卢少勇, 刘学欣, 李珂, 高硕 晗, 贾建丽, 杨光亚 (2016). 模拟生态种植槽去除雨水 径流中的磷. 环境工程学报, 10, 3434-3438.], Kubilay N, Losno R, Luo C, Maenhaut U McGee KA, Okin GS, Siefert RL Tsukuda.

Mahowald N, Jickells TD, Baker AR, Artaxo P, Benitez-Nelson CR, Bergametti G, Bond TC, Chen Y, Cohen DD, Herut B, Kubilay N, Losno R, Luo C, Maenhaut W, McGee KA, Okin GS, Siefert RL, Tsukud S (2008). Global distribution of atmospheric phosphorus sources, concentrations and deposition rates, and anthropogenic impacts. Global Biogeochemical Cycles, 22, GB4026. DOI: 10.1029/2008GB003240.

Mcgroddy ME, Daufresne T, Hedin LO (2004). Scaling of $\mathrm{C}: \mathrm{N}: \mathrm{P}$ stoichiometry in forests worldwide: Implications of terrestrial Redfield-type ratios. Ecology, 85, 2390-2401.

Meir P, Grace J, Miranda AC (2001). Leaf respiration in two tropical rainforests: Constraints on physiology by phosphorus, nitrogen and temperature. Functional Ecology, 15, 378-387.

Monteith JL, Moss CJ (1977). Climate and the efficiency of crop production in Britain. Philosophical Transactions of the Royal Society B: Biological Sciences, 281, 277-294.

Newman EI (1995). Phosphorus inputs to terrestrial ecosystems. Journal of Ecology, 83, 713-726.

Niu YF, Chai RS, Jin GL, Wang H, Tang CX, Zhang YS (2013). Responses of root architecture development to low phosphorus availability: A review. Annals of Botany, 112, 391-408.

Norby RJ, Gu L, Haworth IC, Jensen AM, Turner BL, Walker AP, Warren JM, Weston DJ, Xu C, Winter K (2017). Informing models through empirical relationships between foliar phosphorus, nitrogen and photosynthesis across 
diverse woody species in tropical forests of Panama. New Phytologist, 215, 1425-1437.

Norby RJ, de Kauwe MG, Domingues TF, Duursma RA, Ellsworth DS, Goll DS, Lapola DM, Luus KA, Mackenzie AR, Medlyn BE, Pavlick R, Ramming A, Smith B, Thomas R, Thonicke K, Walker AP, Yang X, Zaehle S (2015). Model-data synthesis for the next generation of forest free-air $\mathrm{CO}_{2}$ enrichment (FACE) experiments. New Phytologist, 209, 17-28.

Norby RJ, Warren JM, Iversen CM, Medlyn BE, McMurtrie $\mathrm{RE}$ (2010). $\mathrm{CO}_{2}$ enhancement of forest productivity constrained by limited nitrogen availability. Proceedings of the National Academy of Sciences of the United States of America, 107, 19368-19373.

Peñuelas J, Asensio D, Tholl D, Wenke K, Rosenkranz M, Piechulla B, Schnitzler JP (2014). Biogenic volatile emissions from the soil. Plant, Cell \& Environment, 37, 1866-1891.

Pierrou U (1976). The global phosphorus cycle. Ecological Bulletins, 48(22), 75-88.

Plaxton WC, Podestá FE (2006). The functional organization and control of plant respiration. Critical Reviews in Plant Sciences, 25, 159-198.

Pote DH, Daniel TC (1996). Relating extractable soil phosphorus to phosphorus losses in runoff. Soil Science Society of America Journal, 60, 855-859.

Reed SC, Yang X, Thornton PE (2015). Incorporating phosphorus cycling into global modeling efforts: A worthwhile, tractable endeavor. New Phytologist, 208, 324-329.

Reich PB, Oleksyn J (2004). Global patterns of plant leaf N and $\mathrm{P}$ in relation to temperature and latitude. Proceedings of the National Academy of Sciences of the United States of America, 101, 11001-11006.

Scott JT, Condron LM (2005). Short term effects of radiata pine and selected pasture species on soil organic phosphorus mineralisation. Plant and Soil, 266, 153-163.

Slot M, Rey-Sánchez C, Winter K, Kitajima K (2014). Trait-based scaling of temperature-dependent foliar respiration in a species-rich tropical forest canopy. Functional Ecology, 28, 1074-1086.

Smil V (2000). Phosphorus in the environment: Natural flows and human interferences. Annual Review of Energy and the Environment, 25, 53-88.

Spohn M, Zavišić A, Nassal P, Bergkemper F, Schulz S, Marhan S, Schloter M, Kandeler E, Polle A (2018). Temporal variations of phosphorus uptake by soil microbial biomass and young beech trees in two forest soils with contrasting phosphorus stocks. Soil Biology \& Biochemistry, 117, 191-202.

Stitt M, Hurry V (2002). A plant for all seasons: Alterations in photosynthetic carbon metabolism during cold acclimation in Arabidopsis. Current Opinion in Plant Biology, 5,
199-206.

Sun Y, Peng SS, Goll DS, Ciais P, Guenet B, Guimberteau M, Hinsinger P, Janssens IA, Peñuelas J, Piao SL, Poulter B, Violette A, Yang XJ, Yin Y, Zeng H (2017). Diagnosing phosphorus limitations in natural terrestrial ecosystems in carbon cycle models. Earth's Future, 5, 730-749.

Thomas DS, Montagu KD, Conroy JP (2006). Leaf inorganic phosphorus as a potential indicator of phosphorus status, photosynthesis and growth of Eucalyptus grandis seedlings. Forest Ecology and Management, 223, 267-274.

Turner BL, Mahieu N, Condron LM (2003). Phosphorus-31 nuclear magnetic resonance spectral assignments of phosphorus compounds in soil $\mathrm{NaOH}-\mathrm{EDTA}$ extracts. Soil Science Society of America Journal, 67, 497-510.

Ushio M, Fujiki Y, Hidaka A, Kitayama K (2015). Linkage of root physiology and morphology as an adaptation to soil phosphorus impoverishment in tropical montane forests. Functional Ecology, 29, 1235-1245.

van Wijk MT, Williams M, Gough L, Hobbie SE, Shaver GR (2003). Luxury consumption of soil nutrients: A possible competitive strategy in above-ground and below-ground biomass allocation and root morphology for slow-growing arctic vegetation? Journal of Ecology, 91, 664-676.

Wan S, Hui D, Wallace L, Luo Y (2005). Direct and indirect effects of experimental warming on ecosystem carbon processes in a tallgrass prairie. Global Biogeochemical Cycles, 19, GB2014. DOI: 10.1029/2004GB002315.

Wang F, Song MH, Huang M, Zhang JS (2014). The spatial distribution of soil nutrients and the controlling factors of temperate forest and steppe in northeastern China. Ecology and Environmental Sciences, 23, 1280-1285. [王芳, 宋明 华, 黄玫, 张甲珅 (2014). 东北北部温带森林和干草地 土壤养分分布及影响因素. 生态环境学报, 23，12801285.]

Wang R, Balkanski Y, Boucher O, Ciais P, Peñuelas J, Tao S (2015). Significant contribution of combustion-related emissions to the atmospheric phosphorus budget. Nature Geoscience, 8, 48-54.

Wang R, Sun QQ, Wang Y, Liu QF, Du LL, Zhao M, Gao X, $\mathrm{Hu}$ YX, Guo SL (2017). Temperature sensitivity of soil respiration: Synthetic effects of nitrogen and phosphorus fertilization on Chinese Loess Plateau. Science of the Total Environment, 574, 1665-1673.

Wang YP, Houlton BZ, Field CB (2007). A model of biogeochemical cycles of carbon, nitrogen, and phosphorus including symbiotic nitrogen fixation and phosphatase production. Global Biogeochemical Cycles, 21, GB1018. DOI: 10.1029/2006GB002797.

Wang YP, Law RM, Pak B (2010). A global model of carbon, nitrogen and phosphorus cycles for the terrestrial biosphere. Biogeosciences, 7, 2261-2282.

Yan XF, Liu YY, Guo QW, Huang DW (2016). Nitrogen and

www.plant-ecology.com 
phosphorus elimination by composited aggregate Bahia grass-planting concrete. Chinese Journal of Environmental Engineering, 10, 1171-1176. [严雄风, 刘迎云, 虢清伟, 黄大伟 (2016). 组合骨料型百喜草植生混凝土去除氮 磷. 环境工程学报, 10, 1171-1176.]

Yan XL, Liao H, Ge ZY, Luo XW (2000). Root architectural characteristics and phosphorus acquisition efficiency in plants. Chinese Bulletin of Botany, 21, 511-519. [严小龙, 廖红, 戈振扬, 罗锡文 (2000). 植物根构型特性与磷吸 收效率. 植物学通报, 21, 511-519.]

Yang X, Thornton PE, Ricciuto DM, Post WM (2014). The role of phosphorus dynamics in tropical forests-A modeling study using CLM-CNP. Biogeosciences, 11, 1667-1681.

Zhang DS, Zhang CC, Tang XY, Li HG, Zhang FS, Rengel Z, Whalley WR, Davies WJ, Shen JB (2016). Increased soil phosphorus availability induced by faba bean root exudation stimulates root growth and phosphorus uptake in neighbouring maize. New Phytologist, 209, 823-831.

Zhang Q, Wang YP, Matear RJ, Pitman AJ, Dai YJ (2014). Nitrogen and phosphorous limitations significantly reduce future allowable $\mathrm{CO}_{2}$ emissions. Geophysical Research Letters, 41, 632-637.

Zhang YM, Zhou GS (2012). Primary simulation on the response of leaf maximum carboxylation rate to multiple environmental factors. Chinese Science Bulletin, 57, 1112-1118. [张彦敏, 周广胜 (2012). 植物叶片最大羧 化速率对多因子响应的模拟. 科学通报, 57, 1112-1118.]

Zhao Q, Zeng DH (2005). Phosphorus cycling in terrestrial ecosystems and its controlling factors. Acta Phytoecologica Sinica, 29, 153-163. [赵琼, 曾德慧 (2005). 陆地 生态系统磷素循环及其影响因素. 植物生态学报, 29 , 153-163.]

Zhou ZH, Wang CK (2016). Changes of the relationships between soil and microbes in carbon, nitrogen and phosphorus stoichiometry during ecosystem succession. Chinese Journal of Plant Ecology, 40, 1257-1266. [周正虎, 王传 宽 (2016). 生态系统演替过程中土壤与微生物碳氮磷 化学计量关系的变化. 植物生态学报, 40, 1257-1266.]

责任编委: 杜 盛 责任编辑: 李 敏 实习编辑: 赵 航 